\title{
O NEO-ALAGOAS NAS BACIAS DO CEARÁ, ARARIPE E POTIGUAR (BRASIL): CARACTERIZAÇÃO ESTRATIGRÁFICA E PALEOAMBIENTAL
}

\author{
ARMANDO TERUO HASHIMOTO*, CIRO JORGE APPI** , \\ ANA LÚCIA SOLDAN*** e JOSÉ ROBERTO CERQUEIRA***
}

\begin{abstract}
The stratigraphic analysis of the Alagoas Stage (local stage) in Ceará Basin allows the investigated interval to be divided into two parts, with distinctive tectono-sedimentary and paleobiological features. The lower part shows non-marine environmental character and the upper part, informally named Aptian-Albian transition, contains marine organisms in certain levels. These records would represent the first phases of a primitive equatorial Atlantic Ocean in the area. The Trairi Beds at the top of the continental section were deposited in an environmental complex which includes a marginal sabkha, reflecting cyclical conditions of extreme aridity or humidity. Similar events have occurred in the Potiguar Basin with the Ponta do Tubarão Beds and in Araripe Basin with the deposition of thinny calcareous and shale layers, here informally named "Batateira Beds". These environmental conditions provided an excellent setting for preservation of organic matter due to cyclical anoxic processes. The observation that the organic extracts from shales of all these strata are similar supports the hypothesis of correspondence of these events.
\end{abstract}

INTRODUÇÃO Com vistas à análise estratigráfica do Andar Alagoas, a Petrobrás vem desenvolvendo estudos programados para cobrir as principais bacias da margem continental brasileira.

Como ponto de partida, face às necessidades exploratórias da companhia, escolheu-se a Bacia do Ceará, onde, verificando-se a dificuldade de subdivisão biocronoestratigráfica deste andar, adotou-se um recurso que consiste no reconhecimento e marcação de eventos geológicos importantes na bacia, constituindo não uma estratigrafia formal mas uma "estratigrafia por eventos".

MÉTODOS Foram realizadas análises sedimentológicas e bioestratigráficas (palinologia, foraminíferos e ostracodes) de amostras de calha e testemunhos da Bacia do Ceará (Sub-bacia de Mundaú), associadas aos estudos de perfis elétricos e seções sísmicas.

Face à dificuldade de subdivisão biocronoestratigráfica desta parte da seção, utilizou-se o reconhecimento de eventos geológicos importantes, tais como transgressões e regressões, dessecações, discordâncias erosivas e modificações estruturais, constituindo uma "estratigrafia por eventos", que permitiu o estabelecimento do arcabouço tectono-sedimentar da bacia, o qual se encontra mais detalhado na $A n \dot{a}$ lise estratigráfica do Andar Alagoas na Bacia do Ceará (Della Fávera et al. 1984). Sob este prisma, da não-utilização dos conceitos de uma estratigrafia formal, a culminância dos eventos transgressivos, um fenômeno regional de controle climático, constitui um elemento de correlação que pode ser buscado nas bacias, independentemente do contexto tectônico.

Foram, também, realizadas análises geoquímicas de cromatografia em fase gasosa das parafinas dos extratos orgânicos, reflectância de vitrinitas (Ro), análises isotópicas do carbono ( $\delta^{13} \mathrm{C}$ padrão $\left.\mathrm{PDB}\right)$ e análise de biomarcadores (esteranos e terpanos).

$\mathrm{O}$ reconhecimento de eventos com as mesmas caracteristicas sedimentares e biológicas em Estudo faciológico da Chapada do Araripe (Appi et al. 1985), associado aos resultados geoquímicos obtidos em Avaliação geoquímica $\mathrm{da} B \mathrm{Ba}$ cia do Ceará (Mello et al. 1984b), na Avaliação geoquímica da plataforma continental da Bacia Potiguar (Mello et al. 1984a) e em amostras de afloramentos da Chapada do Araripe, possibilitaram a correlação dessas bacias (Fig. 1) e evidenciaram certos fenômenos de amplitude regional.

RESULTADOS OBTIDOS Bacia do Ceará Na Bacia do Ceará (Quadro 1), o Andar Alagoas, em termos de características sedimentares e paleobiológicas, pode ser dividido em duas partes: a inferior, com características ambientais continentais, e a parte superior, denominada informalmente transição Aptiano-Albiano, que contém níveis com organismos marinhos (foraminíferos quitinosos, dinoflagelados, foraminíferos planctônicos e bentônicos etc.).

Em termos de comportamento tectono-sedimentar, a seção continental pode ser subdividida em duas porções, acima e abaixo do marco 90 (marco elétrico). A porção inferior caracteriza-se por uma sedimentação muito compartimentada (Fig. 2) de tal maneira que os depósitos se apresentam irregulares e com pequena extensão lateral; apenas os grandes eventos, como culminância de transgressões e modificações estruturais, são correlacionáveis regionalmente. A porção superior, pelo fato de ter sido acompanhada de condições tectônicas mais suaves, mostra uma razoável regularidade nos depósitos, o que permite correlações mais detalhadas e por distâncias mais longas.

No topo da seção continental, que na área investigada está situada no topo da biozona P-270, depositaram-se as Camadas Trairi, que são intercalações de calcários e folhe-

\footnotetext{
* Centro de Pesquisas da Petrobrás (Cenpes), Divisão de Exploração, Setor de Bioestratigrafia e Paleoecologia. Cidade Universitária, Quadra 7, Prédio 20, Ilha do Fundão, CEP 21910, Rio de Janeiro, RJ, Brasil

** Idem, Setor de Geologia.

*** Idem, Setor de Geoquímica.
} 
lhos, depositados em um complexo ambiental do qual o

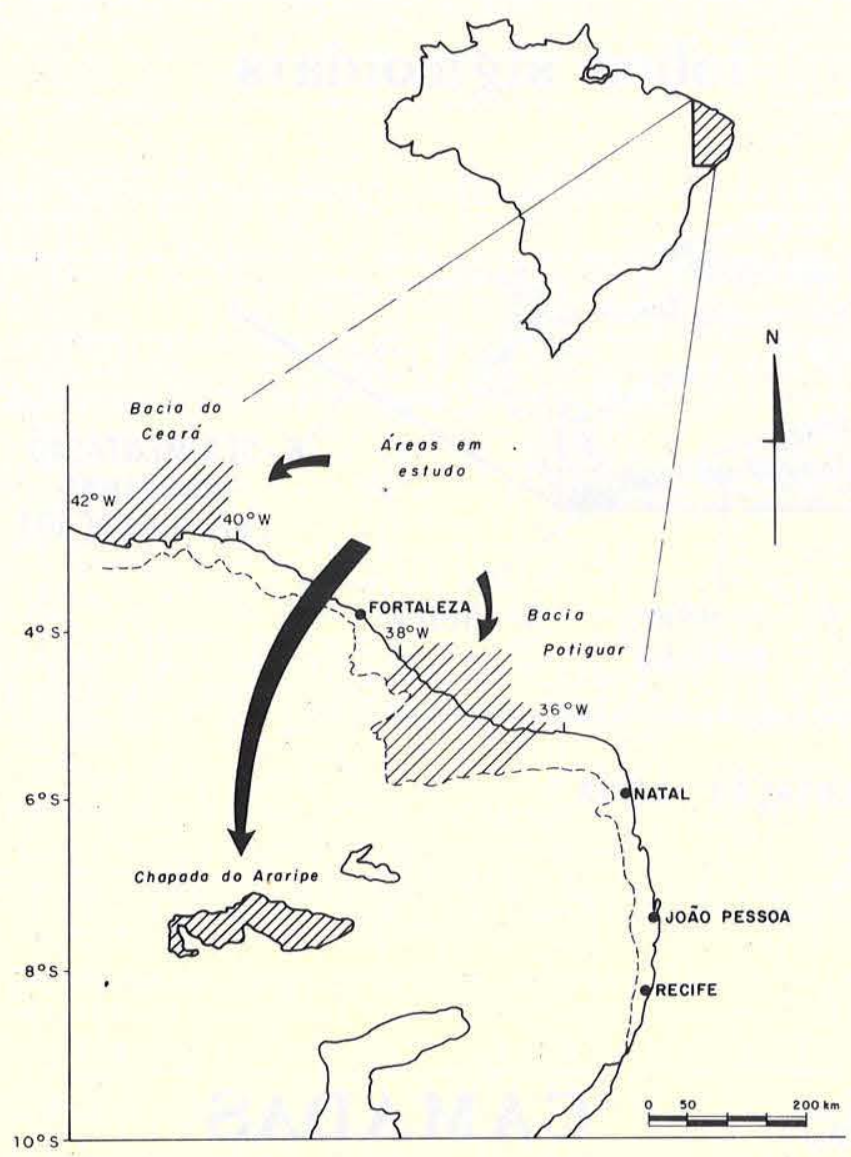

Figura 1 - Mapa de localização

sabkha marginal faz parte (Fig. 3), refletindo condições alternadas de extrema aridez e umidade, que propiciam excelentes condições de preservação de matéria orgânica.

A cromatografia em fase gasosa das parafinas dos extra tos orgânicos mostra a razão pristano/fitano $(\mathrm{P} / \mathrm{F})$ menor que 1. A razão entre os isótopos estáveis do carbono $\left(\delta^{13} \mathrm{C}\right)$ apresenta o valor de $-25,4 \%$ o.

A seção denominada zona de transição Aptiano-Albiano apresenta as primeiras evidências de influência marinha. Sua espessura é extremamente variável e encontra-se bioestratigraficamente indefinida, muitas vezes pela presença de possíveis elementos retrabalhados de biozonas mais velhas. Representa o registro sedimentar de um novo pulso tectônico que se estabeleceu na bacia, modificando as condições de relativa estabilidade preexistentes.

Bacia do Araripe Na Bacia do Araripe, a partir da seqüência sedimentar exposta no Rio da Batateira, foi possível o reconhecimento de eventos correlacionáveis com a Bacia do Ceará (Fig. 4).

A culminância do primeiro evento transgressivo está representada por uma camada de folhelho betuminoso subjacente a uma camada calcária com feições de exposição. Tais camadas são aqui, informalmente, denominadas "Camadas Batateira" e são correlacionáveis às Camadas Trairi.
A razão pristano/fitano $(\mathrm{P} / \mathrm{F})$ desses extratos orgânicos é menor que 1 e a razão isotópica do carbono $\left(\delta^{13} \mathrm{C}\right)$ é de $-26,6 \%$.

A culminância do segundo evento transgressivo, mais as seções evaporíticas, coincide com outro intervalo bastante característico na Bacia do Ceará - a zona de transição Aptiano-Albiano - onde desaparecem os pólens da biozona P-270.

Bacia Potiguar Quanto à Bacia Potiguar, verifica-se, segundo os dados existentes em relatórios de poços (vários autores), que as Camadas Ponta do Tubarão, representadas por calcários e folhelhos depositados em condições alternadas de aridez e umidade, correlacionam-se muito bem com as Camadas Trairi (Fig. 5).

A razão pristano/fitano é menor que 1 , com valor de $\delta^{13} \mathrm{C}$ de $-25,2 \%$.

A zona de transição Aptiano-Albiano encontra correspondência com sedimentos dos membros Galinhos e Aracati da Formação Alagamar.

DISCUSSÃO DOS RESULTADOS A "estratigrafia por eventos" utilizada no estudo da Bacia do Ceará evidencia certos fenômenos que por suas próprias características indicam

Quadro 1 - Sumário estratigráfico (adaptado de Della Fávera et al. 1984)

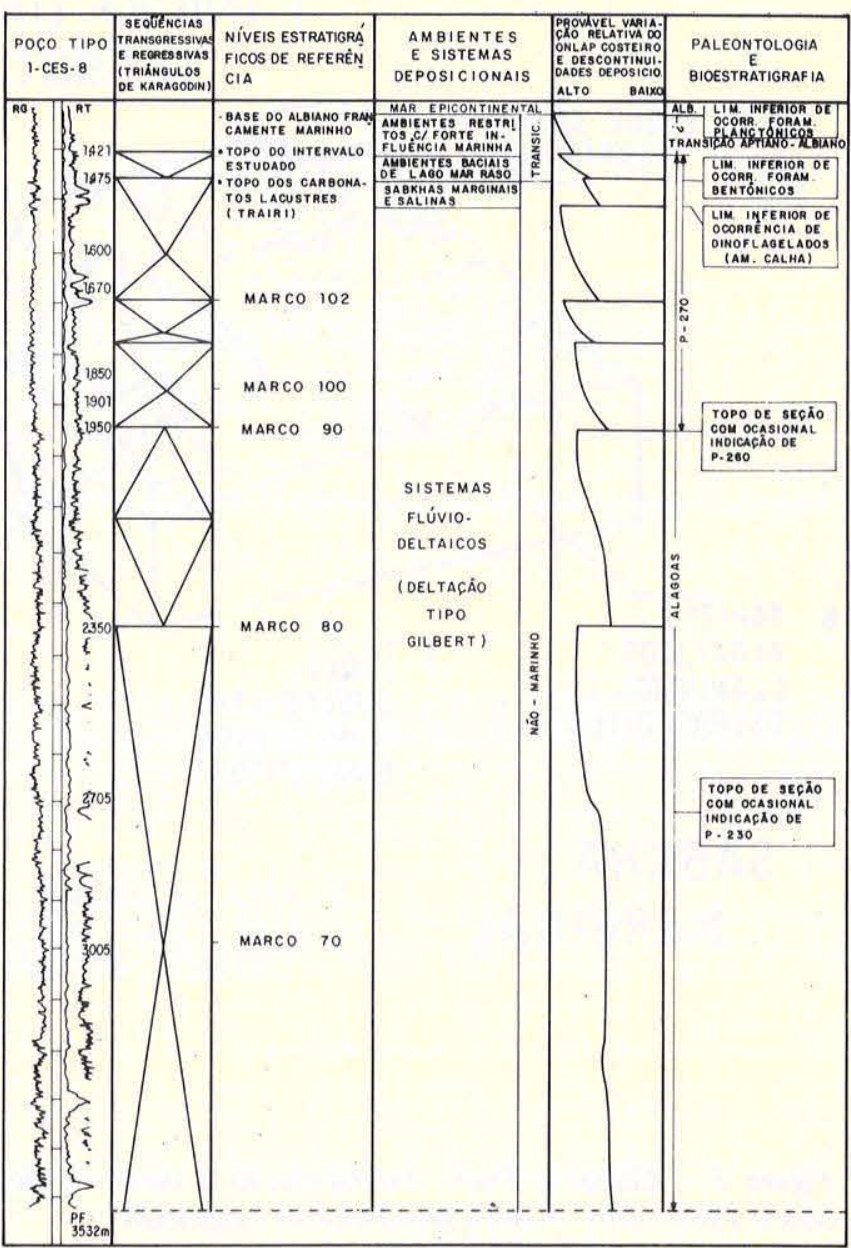




\section{FÁCIES "DELTAICAS"}

\section{lobos sigmoidais}

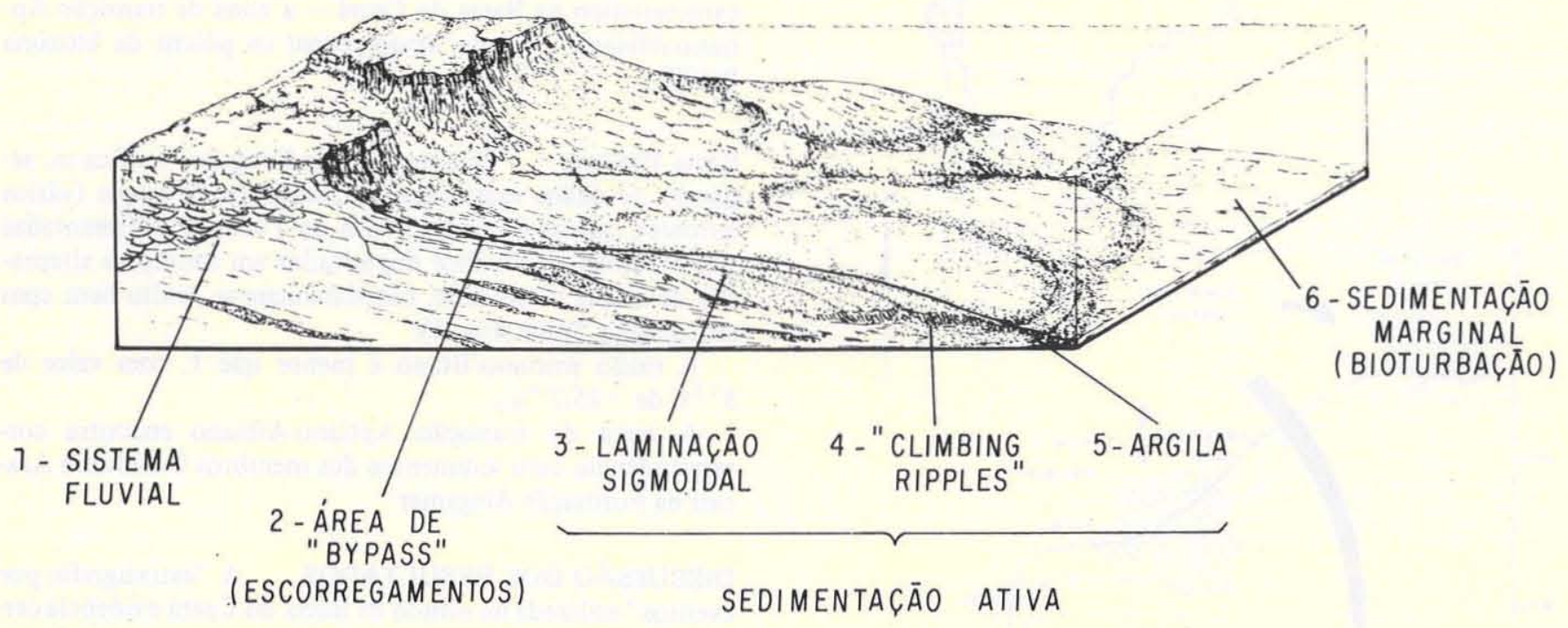

Figura 2 - Fácies “deltaicas” (adaptado de Della Fávera et al. 1984)

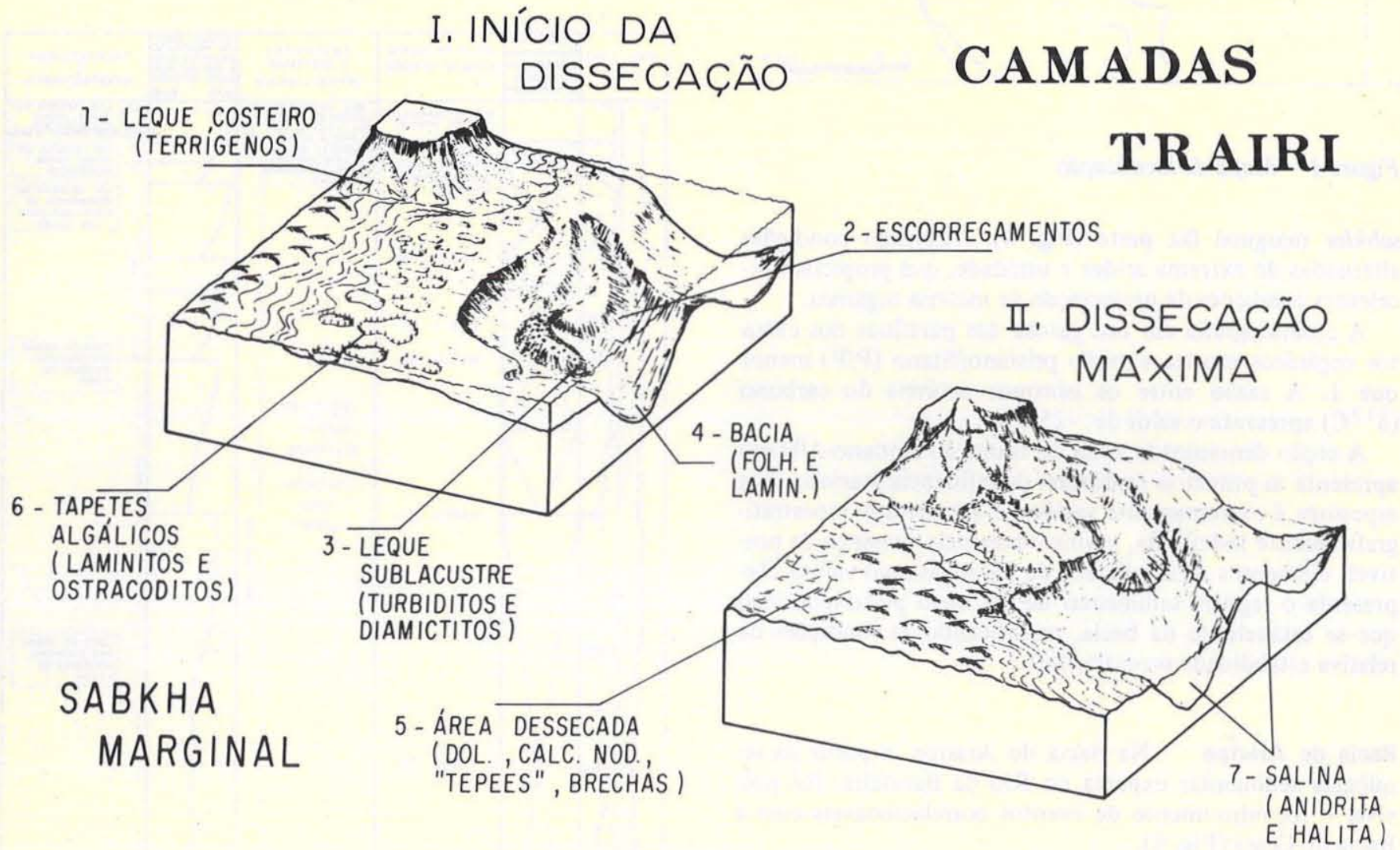

Figura 3 - Camadas Trairi. Interpretação a partir de descrições de testemunho do 7-XA-14-CES (por Barrocas, Castro \& Souza 1983). Obs. : Relevo grandemente exagerado 


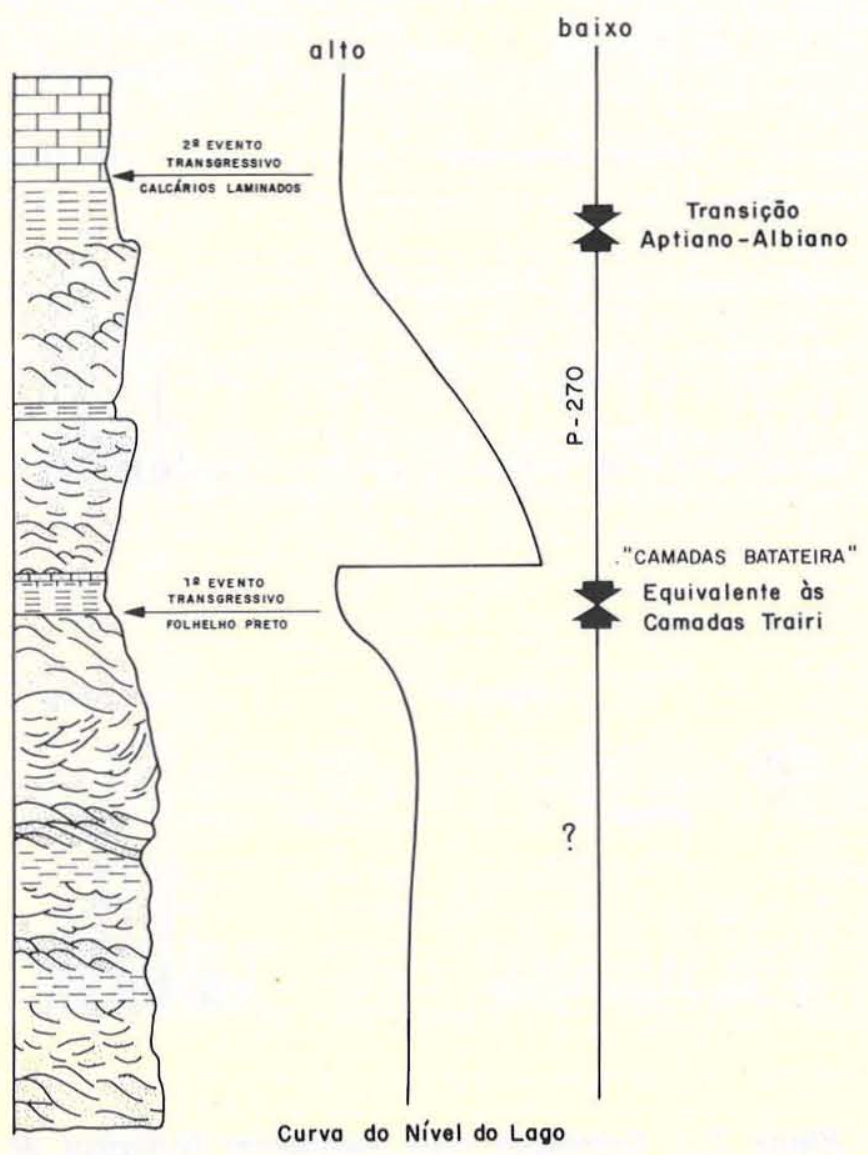

ESCALA $-1: 500$

ALTURA APROX. $-100 \mathrm{~m}$

Figura 4 - Bacia do Araripe - Perfil esquemático do Rio da Batateira (adaptado de Appi et al. 1985)

alterações geológico-climáticas de âmbito regional. Duas dessas alterações são bastantes conspícuas. A primeira é representada pela alternância de folhelhos ricos em matéria orgânica e calcários com feições de exposição, indicando alternância de fases úmidas com outras de extrema aridez. Sistematicamente, estas camadas, denominadas Camadas Trairi, são posicionadas no Andar Alagoas e pertencem à biozona Sergipea variverrucata (P-270).

A segunda alteração muito conspícua apresenta uma seção com características ambientais transicionais, onde os primeiros registros de organismos marinhos são encontrados e representariam as primeiras fases do Atlântico equatorial primitivo nesta área. Embora bioestratigraficamente indefinida, tem, entretanto, sua posição estratigráfica bem marca$\mathrm{da}$, situando-se sempre entre as camadas continentais sotopostas e sedimentos marinhos albianos (ou mais novos) sobrepostos.

Comparando-se esses resultados com dados provenientes das bacias Potiguar e do Araripe, verifica-se que eventos semelhantes lá ocorreram e tanto suas características sedimentológicas como paleobiológicas apresentam uma correspondência perfeita.

Muito importantes, principalmente pela forma independente com que foram conduzidos, os resultados geoquímicos vêm corroborar a tese da correspondência desses eventos geológico-climáticos.
Os extratos orgânicos obtidos dos folhelhos das camadas Trairi, "Batateira" e Ponta do Tubarão, nas bacias do Ceará, do Araripe e Potiguar são correlacionáveis entre si, evidenciando semelhança nas condições anóxicas reinantes à época de deposição.

A cromatografia em fase gasosa das parafinas desses extratos evidencia razão pristano/fitano menor que 1 nos três casos. Observa-se que a distribuição de parafinas normais apresenta variações em suas proporções, de acordo com o estágio de maturação atingido, como evidenciam os valores de porcentagem de reflectância de vitrinitas (Ro).

Da mesma forma, deve-se observar, nos cromatogramas das seqüências estudadas, que a proporção de marcadores biológicos na Bacia do Araripe é bem superior à da Potiguar e quase ausente na do Ceará, o que reflete mais uma vez o efeito de diferentes estágios de maturação nestes sedimentos (Fig. 6).

A razão entre os isótopos estáveis do carbono situa-se entre $-25,2 \%$ o na Bacia Potiguar e $-26,6 \%$ o na do Araripe, com valor intermediário de $-25,4 \%$ o na do Ceará. Esses valores indicam que os ambientes deposicionais nos três casos estudados continham água salgada.

Entre os biomarcadores, observa-se que, nas bacias do Ceará e Potiguar, os fragmentogramas possuem na família dos triterpanos, proporções semelhantes entre o gamacerano e o hopano. No grupo dos esteranos, destaca-se a elevada proporção do colestano (Fig. 7). Essas características têm sido observadas em seções evaporíticas de diversas bacias analisadas pelo Setor de Geoquímica da Petrobrás.

Esses dados, em conjunto, indicam que a paleobiomassa orgânica presente nesta seqüência, bem como as condições

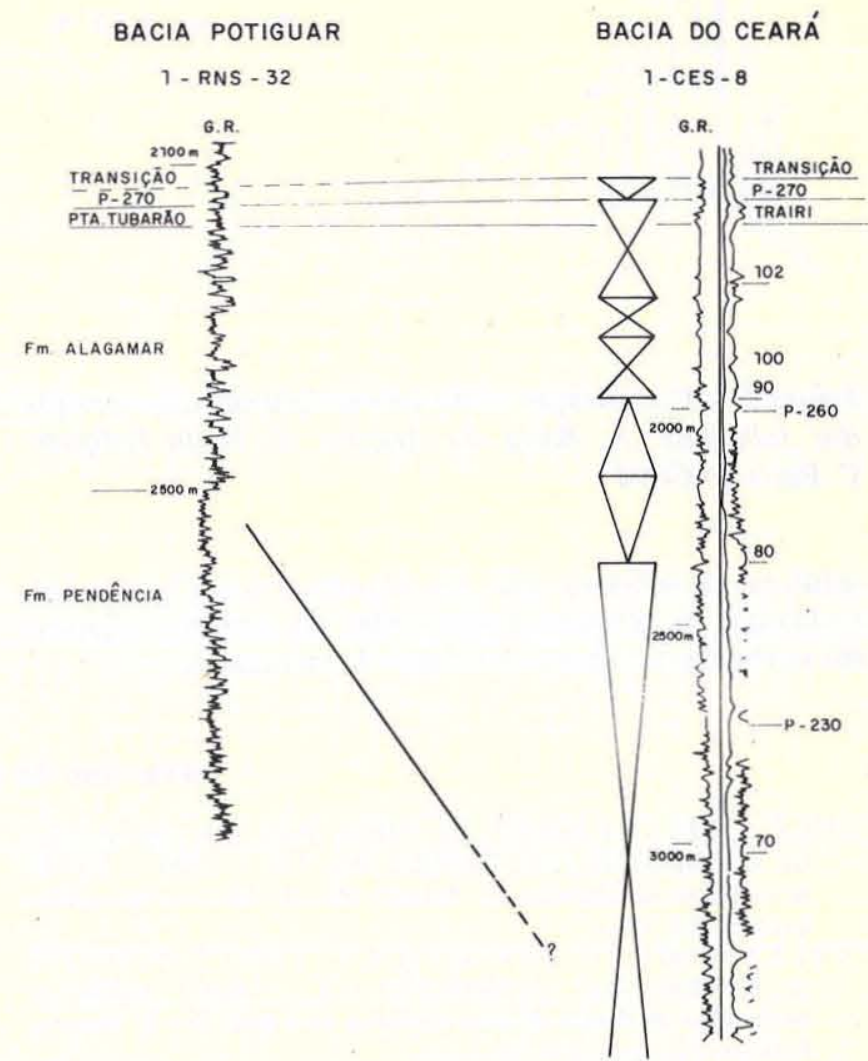

Figura 5 - Diagrama de correlação (adaptado de Della Fávera et al. 1984) 


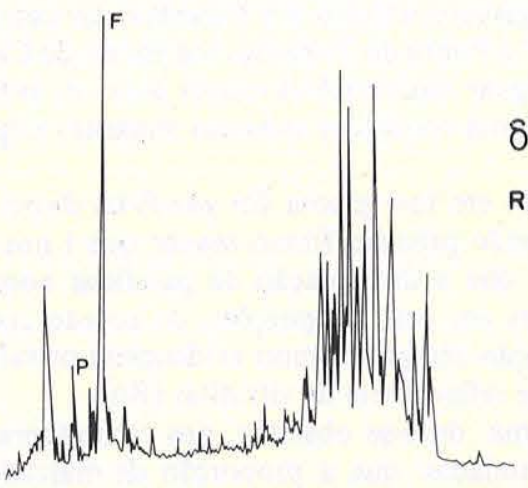

(A)

$\delta^{13} \mathrm{C}=-26,6 \%$

$R_{0}=0,33 \%$

$P=$ PRISTANO

$F=$ FITANO

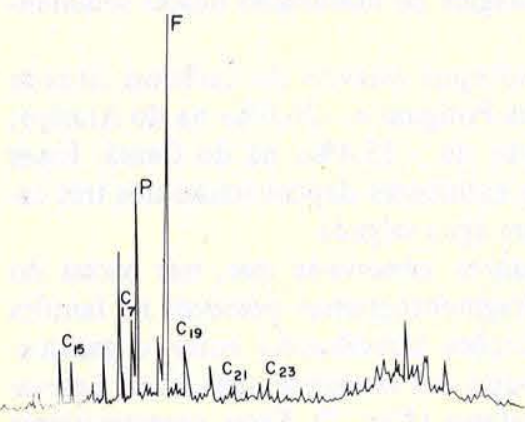

(B)

$\delta^{13} \mathrm{C}=-25,2 \%$ 。

$R_{0}=0,53 \%$

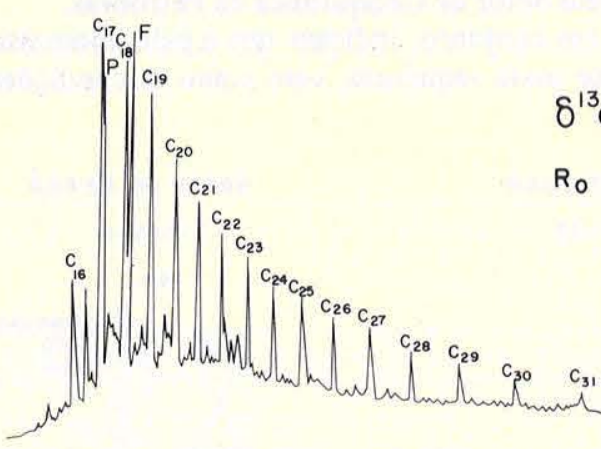

Figura 6 - Comparação entre cromatogramas dos extratos dos folhelhos: A. Bacia do Araripe; B. Bacia Potiguar; C. Bacia do Ceará

redutoras de sedimentação, são semelhantes nas três bacias estudadas. As diferenças observadas no material orgânico são atribuídas aos diversos estágios de maturação.
TRITERPANOS
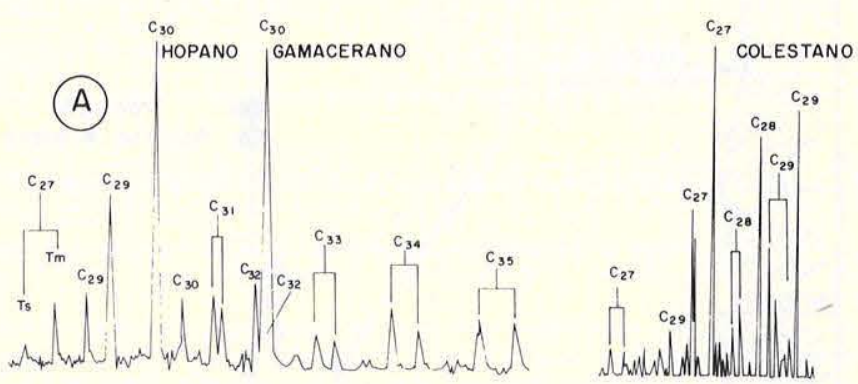

Figura 7 - Correlação entre marcadores biológicos de extratos orgânicos dos folhelhos: A. Bacia Potiguar e B. Bacia do Ceará

CONCLUSÕES As análises de dados sedimentológicos, paleobiológicos e geoquímicos revelam que as Camadas Trairi (Bacia do Ceará), Camadas Ponta do Tubarão (Bacia Potiguar) e as aqui denominadas "Camadas Batateira" (Bacia do Araripe) depositaram-se sob condições geológico-climáticas similares, o que parece ter sido resultado de um evento regional que ocorreu em todo o Nordeste brasileiro.

A natureza dessas condições atuantes à época de sedimentação pode ser reconhecida nas intercalações de camadas de calcários e folhelhos, indicativas de alternância de climas áridos e úmidos.

Outro evento bastante característico é representado pelas primeiras evidências biológicas comprovadamente marinhas, que indicam as primeiras fases de um Atlântico equatorial primitivo na área, durante a fase de transição Aptiano-Albiano.

\section{REFERÊNCIAS BIBLIOGRÁFICAS}

APPI, C.J.; DELLA FĀVERA, J.C.; MEDEIROS, R.A.; BEURLEN, G.; HASHIMOTO, A.T.; CERQUEIRA, J.R. - 1985 - Estudo faciológico da Chapada do Araripe. Rio de Janeiro, Petrobrás/ Cenpes (Rel. int.).

DELLA FÁVERA, J.C.; MEDEIROS, R.A.; APPI, C.J.; BEURLEN, G.; VIVIERS, M.C.; HASHIMOTO, A.T. - 1984 - Análise estratigráfica do Andar Alagoas na Bacia do Ceará. Rio de Janeiro, Petrobrás/Cenpes (Rel. int.).

MELLO, M.R.; CERQUEIRA, J.R.; SOLDAN, A.L.; ARARIPE, P.T. - 1984a - Avaliação geoquímica da plataforma continen- tal da Bacia Potiguar. Rio de Janeiro, Petrobrás/Cenpes (Rel. int.).

MELLO, M.R.; SOLDAN, A.L.; CERQUEIRA, J.R.; BELTRAM, C.V. - 1984b - Avaliação geoquímica da Bacia do Ceará. Rio de Janeiro, Petrobrás/Cenpes (Rel. int.).

MANUSCRITO 380

Recebido em 01 de Setembro de 1986 Revisão aceita em 30 de Março de 1987 